\title{
A simple method to compute white dwarf luminosity functions
}

\author{
E. García-Berro ${ }^{1}$, M. Hernanz ${ }^{2}$, J. Isern ${ }^{2}$, G. Chabrier ${ }^{3}$, L. Segretain ${ }^{3}$ and R. Mochkovitch ${ }^{4}$ \\ 1 Departament de Física Aplicada, Universitat Politècnica de Catalunya, Jordi Girona Salgado s/n, Mòdul B-5, \\ Campus Nord, 08034 Barcelona, Spain \\ 2 Centre d'Estudis Avançats de Blanes, C.S.I.C., Camí de Santa Bàrbara, 17300 Blanes, Spain \\ 3 Groupe d'Astrophysique, CNRS, Ecole Normale Supérieure de Lyon, 69394 Lyon Cedex 07, France \\ 4 Institut d'Astrophysique de Paris, CNRS, 98 bis Bd. Arago, 75014 Paris, France
}

Received May 9; accepted October 2, 1995

\begin{abstract}
The luminosity function of white dwarfs can be used to obtain helpful information about the properties of the Galaxy and allows one to discriminate among different models of galactic evolution. In this paper we provide the necessary tools to construct theoretical luminosity functions for any star formation rate, any initial mass function, and any relationship between the mass of the white dwarf and the mass of the progenitor.
\end{abstract}

Key words: white dwarfs — stars: evolution — luminosity function

\section{Introduction}

The comparison between the theoretical and the observational luminosity functions of white dwarfs is a powerful tool to determine some aspects of galactic evolution, such as the history of the star formation rate (Noh \& Scalo 1990; Díaz-Pinto et al. 1994), the age of the disk (Winget et al. 1987; García-Berro et al. 1988a, b; Hernanz et al. 1994), or the properties of the halo (Mochkovitch et al. 1990). Nevertheless, this comparison demands reliable theoretical white dwarf luminosity functions which, in turn, require an accurate white dwarf cooling theory.

One of the most important phenomena occuring during white dwarf evolution is crystallization. Two essential physical processes are associated with solidification, namely a release of latent heat and a chemical fractionation in the mixture, which both provide extra energy sources and lengthen the cooling time of the star. Although the release of the latent heat has been included in the calculations of the energetic balance of cooling white dwarfs for a long time (Van Horn 1968; Lamb \& Van Horn 1975; Iben \& Tutukov 1984; Wood 1992), the effect of chemical fractionation, though energetically dominant, has been usually ignored, essentially because the accurate treatment of such process requires much more complicated calculations. This effect depends on the shape of the phase diagram and on the initial chemical profile of the white dwarf. The most accurate calculation of the properties of arbitrary binary ionic mixtures is that of Segretain \& Chabrier (1993). These authors find that the shape of the phase diagram for the carbon-oxygen binary mixture is of the spindle form and therefore their predic- tion is that some chemical fractionation would occur when crystallization sets in. Although there is little doubt about the shape of the phase diagram, there is still some debate about the question of whether the process of chemical fractionation would operate in real stars with $100 \%$ efficiency. The consequences of this process on the cooling of white dwarfs and on the white dwarf luminosity function have been presented elsewhere (Segretain et al. 1994; Hernanz et al. 1994).

The purpose of this paper is to provide a simple method to construct accurate enough white dwarf luminosity functions to test different theoretical models of galactic evolution.

\section{The luminosity function}

In order to avoid the influence of the initial conditions, which are poorly known, calculations start at luminosities around $L_{0}=10^{-1} L_{\odot}$. In fact, the time necessary to reach such a luminosity is very short. Thus, if the hydrogen envelope is thin enough, nuclear burning contributions can be ignored and the luminosity of the star only arises from the thermal and gravitational energy release. Therefore, the equation of conservation of energy reduces to:

$$
L(t)=-\frac{\mathrm{d} B(t)}{\mathrm{d} t}-L_{\nu}(t)
$$

where $B=E+\Omega$ is the binding energy of the star, $E$ and $\Omega$ are the thermal and gravitational energies, respectively, $L$ is the photon luminosity and $L_{\nu}$ is the neutrino luminosity (Díaz-Pinto et al. 1994; Segretain et al. 1994; Hernanz et 
al. 1994). The luminosity function is given by

$$
\begin{aligned}
n\left(M_{\mathrm{bol}}\right) \propto \int_{M_{\mathrm{i}}(L)}^{M_{\mathrm{s}}} \tau_{\mathrm{cool}}(L, M) & \psi\left(t_{\mathrm{disk}}-t_{\mathrm{cool}}(L, M)\right. \\
& \left.-t_{\mathrm{ms}}(M)\right) \times \phi(M) \mathrm{d} M
\end{aligned}
$$

where $\psi$ is the star formation rate per unit volume, $\phi(M)$ is the initial mass function, $M$ is the mass of the progenitor of the white dwarf, $t_{\text {disk }}$ is the adopted age of the disk, and $t_{\text {cool }}$ is the time required to cool down to a luminosity $L$, given by

$$
t_{\text {cool }}(L, M)=\int_{L_{0}}^{L} \frac{\mathrm{d} B}{L_{\text {tot }}}
$$

where $L_{\text {tot }}=L+L_{\nu}$ is the total luminosity (photon plus neutrino). The characteristic cooling time, $\tau_{\text {cool }}=$ $\mathrm{d} t_{\text {cool }} / \mathrm{d} M_{\text {bol }}$, is computed from

$$
\tau_{\text {cool }}(L, M)=-0.92 \frac{\mathrm{d} B}{\mathrm{~d} T} \frac{\mathrm{d} T}{\mathrm{~d} L} \frac{1}{1+\frac{L_{\nu}}{L}}
$$

$M_{\mathrm{S}}$ is the maximum mass of the progenitor of a carbonoxygen white dwarf. We have chosen $M_{\mathrm{s}}=8 M_{\odot}$ but the results are relatively insensitive to the exact value of $M_{\mathrm{s}}$, $M_{\mathrm{i}}$ is obtained from:

$$
t_{\mathrm{ms}}\left(M_{\mathrm{i}}\right)+t_{\text {cool }}\left(L, M_{\mathrm{i}}\right)=t_{\text {disk }}
$$

The proportionality constant in Eq. (1) can be obtained by fitting the theoretical white dwarf luminosity function to the observations (Liebert et al. 1988).

Note that in Eq. (1) we are using the mass of the progenitor instead of the mass of the white dwarf. Therefore, a relationship linking both masses must be provided. As seen in Eqs. (1) and (2) we also need the lifetime of the progenitor of the white dwarf, $t_{\mathrm{ms}}$, and therefore this must be also provided.

\section{The cooling sequences}

The equations of state used to compute the cooling sequences have been presented in previous papers (Segretain et al. 1994; Chabrier 1993). They allow an accurate treatment of both the liquid and the solid phases, in order to take into account the effects of solidification in the cooling process. Concerning the phase diagram for the carbonoxygen mixture, the most recent results of Segretain \& Chabrier (1993) are adopted.

The initial composition of the white dwarf has been taken from the results of evolutionary calculations of Mazzitelli \& D'Antona (1986), which give chemical composition profiles enriched in oxygen in the central regions (i.e. with an initial stratification) for low mass and moderately massive carbon-oxygen white dwarfs $\left(0.50 M_{\odot} \leq\right.$ $\left.M_{\mathrm{WD}}<0.95 M_{\odot}\right)$, but flat profiles for very massive white

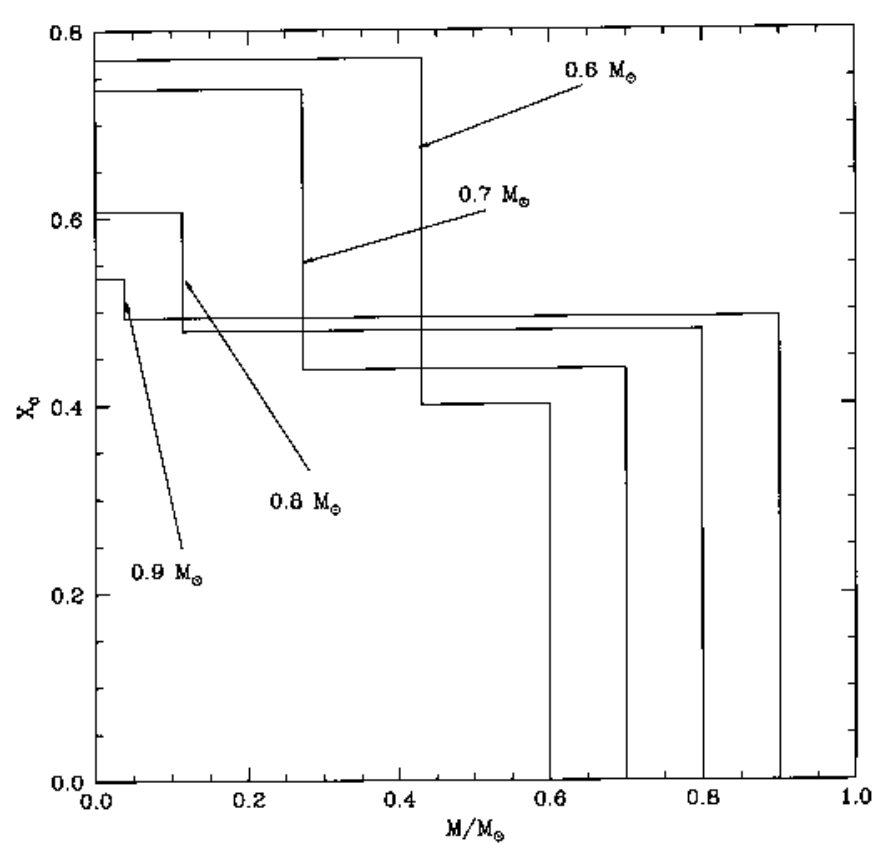

Fig. 1. Initial chemical profiles adopted in our calculations. See text for details

dwarfs $\left(M_{\mathrm{WD}} \geq 0.95 M_{\odot}\right)$. In Fig. 1 we show the oxygen abundance by mass before the onset of crystallization for white dwarfs of masses $M_{\mathrm{WD}}=0.6,0.7,0.8$ and $0.9 M_{\odot}$. Each profile is labelled with its corresponding mass of the white dwarf. The adopted chemical profile for the $0.5 M_{\odot}$ white dwarf is identical to that of the $0.6 M_{\odot}$. Models with initial abundances $X_{\mathrm{C}}=X_{\mathrm{O}}=0.5$ by mass everywhere (i.e. assuming no previous stratification) have also been computed for comparison.

The binding energies before the onset of the solidification process can be fitted as a function of the temperature of the isothermal core by an expression of the form:

$$
B_{\mathrm{L}}(T)=a_{0}+a_{1} T_{6}+a_{2} T_{6}^{2}
$$

where $T_{6}=T / 10^{6}$, whereas once the solidification process sets in at the center of the star, a polynomial of the form:

$$
B_{\mathrm{S}}(T)=b_{0}+b_{2} T_{6}^{2}+b_{3} T_{6}^{3}+b_{4} T_{6}^{4}
$$

is adequate. Note that the coefficient $b_{1}$ does not appear in the last polynomial. The physical reason is that the binding energy must be minimum for $T=0$ (i.e.: zero derivative for $T=0$ ). Tables 1 and 2 display these coefficients for the flat initial profiles and Tables 3 and 4 display the coefficients obtained for the stratified profiles. The temperature at which solidification starts at the center, $T_{6}^{\text {sol }}$, is also shown in Tables 1 and 3 for the flat and stratified initial profiles respectively. It should also be noted that Tables 3 and 4 display only the coefficients of the binding energy for masses in the range 0.5 to 0.9 
Table 1. Coefficients for computing the binding energy in the liquid phase of white dwarfs with initial flat profiles

\begin{tabular}{lcccc}
\hline$M / M_{\odot}$ & $-a_{0}$ & $-a_{1}$ & $-a_{2}$ & $T_{6}^{\text {sol }}$ \\
\hline 0.5 & $2.9715507(0)$ & $-1.4587193(-3)$ & $-3.5844021(-6)$ & $2.9910(0)$ \\
0.6 & $4.6295460(0)$ & $-1.8435664(-3)$ & $-4.8395898(-6)$ & $3.5980(0)$ \\
0.7 & $6.7925117(0)$ & $-2.2350752(-3)$ & $-7.8993080(-6)$ & $4.2960(0)$ \\
0.8 & $9.5518982(0)$ & $-2.6066596(-3)$ & $-1.4068719(-5)$ & $5.1300(0)$ \\
0.9 & $1.3030470(1)$ & $-3.0112621(-3)$ & $-2.4244597(-5)$ & $6.1670(0)$ \\
1.0 & $1.7401409(1)$ & $-3.4380476(-3)$ & $-4.2199735(-5)$ & $7.5270(0)$ \\
1.1 & $2.2933615(1)$ & $-3.8918967(-3)$ & $-7.6319810(-5)$ & $9.4480(0)$ \\
1.2 & $3.0103372(1)$ & $-4.4280643(-3)$ & $-1.5057222(-4)$ & $1.2508(1)$ \\
\hline
\end{tabular}

Table 2. Coefficients for computing the binding energy in the solid phase of white dwarfs with initial flat profiles

\begin{tabular}{lcccc}
\hline$M / M_{\odot}$ & $-b_{0}$ & $-b_{2}$ & $-b_{3}$ & $-b_{4}$ \\
\hline 0.5 & $2.9747514(0)$ & $-5.7186137(-4)$ & $-1.8410747(-4)$ & $4.2190767(-5)$ \\
0.6 & $4.6332738(0)$ & $-5.5586898(-4)$ & $-1.6693878(-4)$ & $3.1900011(-5)$ \\
0.7 & $6.7968535(0)$ & $-5.4829161(-4)$ & $-1.3770878(-4)$ & $2.2219371(-5)$ \\
0.8 & $9.5573322(0)$ & $-5.0129396(-4)$ & $-1.2666263(-4)$ & $1.7129747(-5)$ \\
0.9 & $1.3036813(1)$ & $-4.6202279(-4)$ & $-1.0662122(-4)$ & $1.2000764(-5)$ \\
1.0 & $1.7408682(1)$ & $-4.1944164(-4)$ & $-8.6608900(-5)$ & $7.9636686(-6)$ \\
1.1 & $2.2941927(1)$ & $-3.8466858(-4)$ & $-6.5996663(-5)$ & $4.8064437(-6)$ \\
1.2 & $3.0112426(1)$ & $-3.8027884(-4)$ & $-4.5547433(-5)$ & $2.4752478(-6)$ \\
\hline
\end{tabular}

$M_{\odot}$ since massive white dwarfs do not present stratified initial profiles (Mazzitelli \& D'Antona 1986).

In order to compute $t_{\text {cool }}$ and $\tau_{\text {cool }}$ it is necessary to adopt a relationship between the luminosity of the white dwarf and the temperature of its isothermal core. Of course this relationship depends on the properties (mass, chemical composition, metallicity...) of the adopted envelope for the white dwarf model as well as on the mass of the white dwarf. This relationship must be obtained from a grid of detailed calculations of white dwarf model envelopes. A useful interpolation tool for white dwarfs of any mass is given by the following expression:

$$
\frac{L}{L_{\odot}}=\mathcal{L}(T) \frac{M_{\mathrm{WD}}}{M_{\odot}}
$$

However, given the strongly peaked mass distribution around $0.6 M_{\odot}$ it is enough to obtain $\mathcal{L}(T)$ from a fit of the internal temperature-luminosity relationship for a given model envelope of a $0.6 M_{\odot}$ white dwarf. This scaling law takes into account the leading term in the mass dependence of the luminosity of the white dwarf and has been compared with detailed evolutionary cooling sequences covering a wide range of masses and luminosities (Wood 1990). The accuracy is better than $15 \%$ for any mass of the white dwarf at any temperature in all the cases considered here. Furthermore since the dominant contri- bution to the white dwarf luminosity function is given by white dwarfs of $0.6 M_{\odot}$ this approximation is enough for many purposes.

We have also computed neutrino luminosities for the full range of white dwarf masses assuming an isothermal core and using the most recent neutrino rates (Itoh et al. 1992). For the range of luminosities considered here (corresponding to core temperatures lower than $T=510^{7}$ $\mathrm{K})$ the temperature inversion in the core has disappeared (Wood 1990; Iben \& Tutukov 1984) and this approximation can be regarded as fair enough. The results can be fitted by the following expression

$$
\log \left(L_{\nu}\right)=c_{3}\left(\log T_{6}\right)^{3}+c_{2}\left(\log T_{6}\right)^{2}+c_{1}\left(\log T_{6}\right)+c_{0}
$$

and the corresponding coefficients can be found in Table 5.

It is important to note that the neutrino luminosity, which is the most important energy leak at high temperatures, is completely negligible at low temperatures. This can be seen in Fig. 2 where we have plotted the neutrino luminosity and the (photon) luminosity of a $0.6 M_{\odot}$ white dwarf - see Sect. 4 below - as a function of the temperature. Neutrino cooling affects exclusively the characteristic cooling times (and therefore the shape of the luminosity function) at high luminosity but not the total cooling ages 
Table 3. Coefficients for computing the binding energy in the liquid phase of white dwarfs with stratified initial profiles

\begin{tabular}{lcccc}
\hline$M / M_{\odot}$ & $-a_{0}$ & $-a_{1}$ & $-a_{2}$ & $T_{6}^{\text {sol }}$ \\
\hline 0.5 & $2.9825937(0)$ & $-1.3905197(-3)$ & $-3.7008785(-6)$ & $3.5970(0)$ \\
0.6 & $4.6427282(0)$ & $-1.7657371(-3)$ & $-5.2358353(-6)$ & $4.3200(0)$ \\
0.7 & $6.8013090(0)$ & $-2.1974561(-3)$ & $-8.2180535(-6)$ & $5.0440(0)$ \\
0.8 & $9.5550892(0)$ & $-2.6110333(-3)$ & $-1.4062787(-5)$ & $5.5010(0)$ \\
0.9 & $1.3033837(1)$ & $-3.0175551(-3)$ & $-2.4218299(-5)$ & $6.2250(0)$ \\
\hline
\end{tabular}

Table 4. Coefficients for computing the binding energy in the solid phase of white dwarfs with stratified initial profiles

\begin{tabular}{lccll}
\hline$M / M_{\odot}$ & $-b_{0}$ & $-b_{2}$ & \multicolumn{1}{c}{$-b_{3}$} & \multicolumn{1}{c}{$-b_{4}$} \\
\hline 0.5 & $2.9851888(0)$ & $-8.9418123(-4)$ & $1.3127319(-4)$ & $-8.6461481(-6)$ \\
0.6 & $4.6456954(0)$ & $-1.0954189(-3)$ & $1.9434970(-4)$ & $-1.4963529(-5)$ \\
0.7 & $6.8047140(0)$ & $-8.2026591(-4)$ & $-3.3204417(-5)$ & $1.7816808(-5)$ \\
0.8 & $9.5597076(0)$ & $-2.1078325(-4)$ & $-2.7240249(-4)$ & $3.6082876(-5)$ \\
0.9 & $1.3039971(1)$ & $-4.2881392(-4)$ & $-1.2083527(-4)$ & $1.3666335(-5)$ \\
\hline
\end{tabular}

since its effect is significant only at high luminosities where the cooling ages are extremely short.

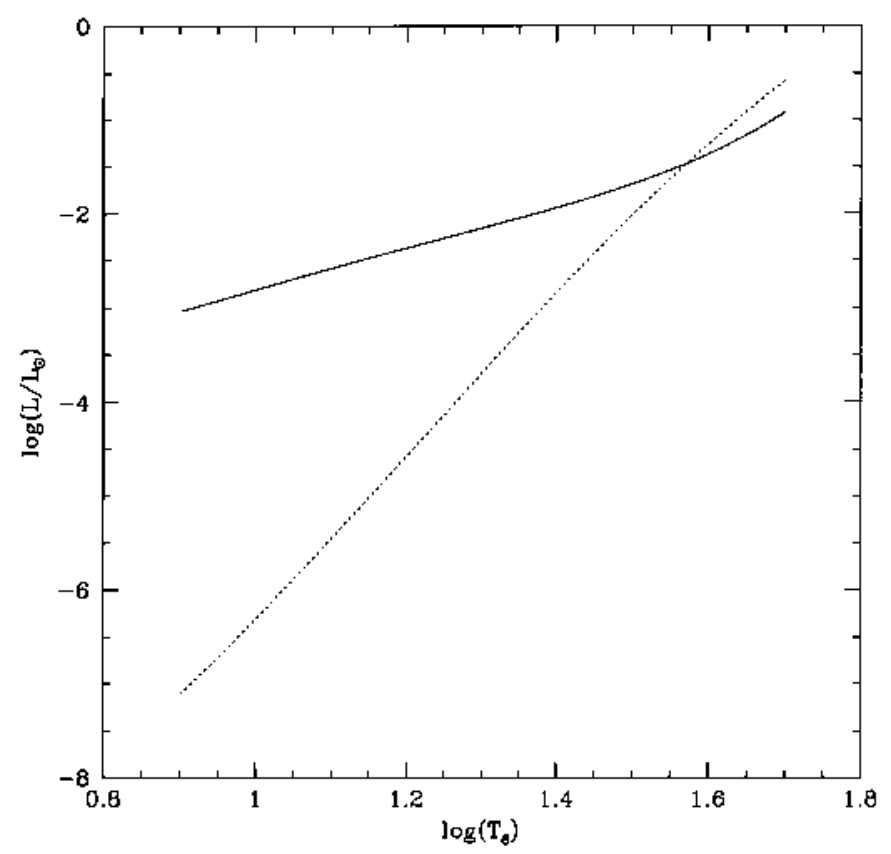

Fig. 2. Neutrino luminosity (dotted line) and (photon) luminosity (solid line) versus temperature for a $0.6 M_{\odot}$ white dwarf

For comparison in Fig. 3 we have plotted as a function of the (photon) luminosity - see Sect. 4 below - the neutrino luminosity for a $0.6 M_{\odot}$ white dwarf computed with the most recent rates of Itoh et al. (1992) - solid line
- the neutrino luminosity obtained using the old rates of Beaudet et al. (1967), for the plasmon, photon and pair neutrino processes, and the rate from De Zotti (1972) for the bremsstrahlung process - short-dashed line - and the neutrino luminosity obtained by Wood \& Winget (1989) - dashed line. As can be seen our approximation is in very good agreement with the results of Wood \& Winget (1989) when the same constitutive physics are considered. This result validates the approximation of assuming an isothermal core. It is also important to stress that the contribution of the bremsstrahlung process to the total rate of neutrino losses is larger when the rates of Itoh et al. (1992) are considered. This is noticeable since it affects the cooling times of the hottest white dwarfs.

\section{Results}

Figure 4 displays an example of the procedure described above. The solid line shows the white dwarf luminosity function computed assuming flat initial profiles of the carbon-oxygen dense plasma and the dotted line is the white dwarf luminosity function assuming a previous stratification of the carbon-oxygen mixture. In both cases we assumed a constant star formation rate and Salpeter's IMF. The adopted age of the galactic disk is 10.5 Gyr in both cases. The mass of the white dwarf progenitors was taken from Weidemann \& Koester (1983). The adopted lifetime in the main sequence for the progenitors of white dwarfs was taken from Iben \& Laughlin (1989). A helium envelope of $M_{\mathrm{He}}=10^{-4} M_{\mathrm{WD}}$ was used (Wood \& Winget 1989). This model envelope can be considered as representative of a non-DA white dwarf atmosphere. The 


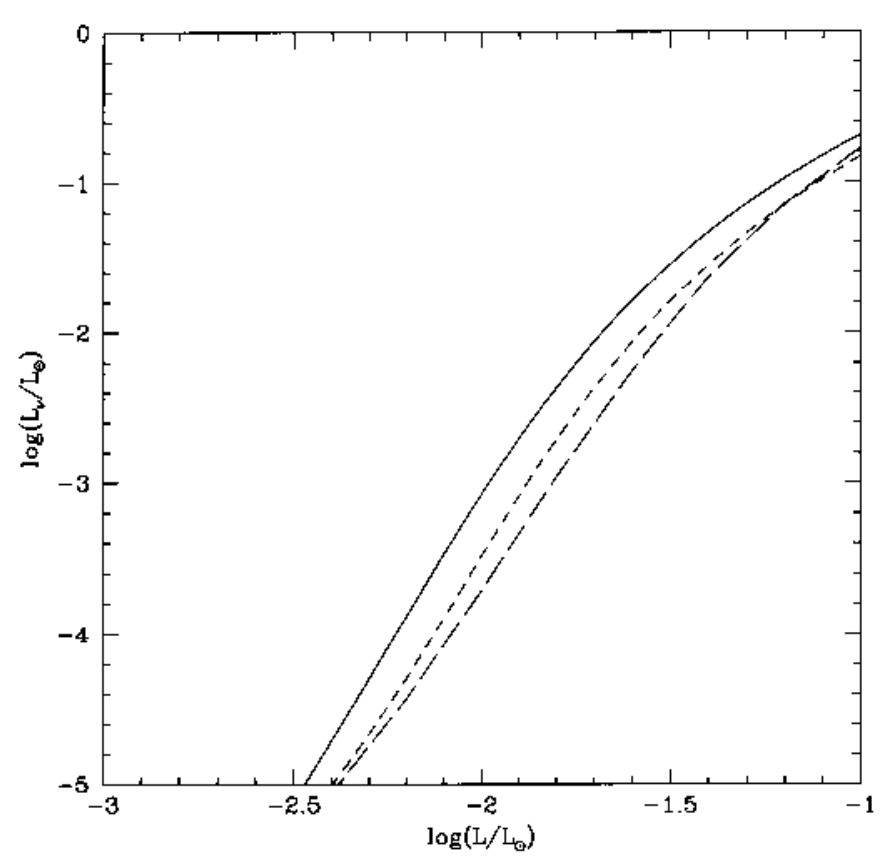

Fig. 3. Neutrino luminosities for different constitutive physics versus (photon) luminosity, see text for details

corresponding fit to the core temperature-luminosity relationship is

$$
\begin{aligned}
\log \mathcal{L}(T)= & 1.98695\left(\log T_{6}\right)^{5}-8.15521\left(\log T_{6}\right)^{4}+ \\
& 11.863\left(\log T_{6}\right)^{3}-6.88515\left(\log T_{6}\right)^{2}+ \\
& 3.16893\left(\log T_{6}\right)-4.56649
\end{aligned}
$$

Table 5. Coefficients for computing neutrino luminosities

\begin{tabular}{lcclc}
\hline$M / M_{\odot}$ & $c_{0}$ & \multicolumn{1}{c}{$c_{1}$} & \multicolumn{1}{c}{$c_{2}$} & $c_{3}$ \\
\hline 0.5 & -10.5994 & -2.90867 & 10.4506 & -3.13602 \\
0.6 & -9.44892 & -5.42330 & 11.8484 & -3.28763 \\
0.7 & -8.59332 & -7.00194 & 12.4250 & -3.23962 \\
0.8 & -7.98463 & -7.86491 & 12.4243 & -3.06462 \\
0.9 & -7.74806 & -7.75171 & 11.6633 & -2.71626 \\
1.0 & -7.21611 & -8.20092 & 11.2940 & -2.47460 \\
1.1 & -12.0398 & 4.46418 & 0.5981730 & 0.392265 \\
1.2 & -11.8746 & 4.71380 & -0.0798432 & 0.619581 \\
\hline
\end{tabular}

As can be seen from Fig. 4 the agreement between the computed white dwarf luminosity function and the observations is good. At high luminosities $\left(\log \left(L / L_{\odot}\right)>-2\right)$ the computed white dwarf luminosity function does not fit the observational data due to the enhanced neutrino

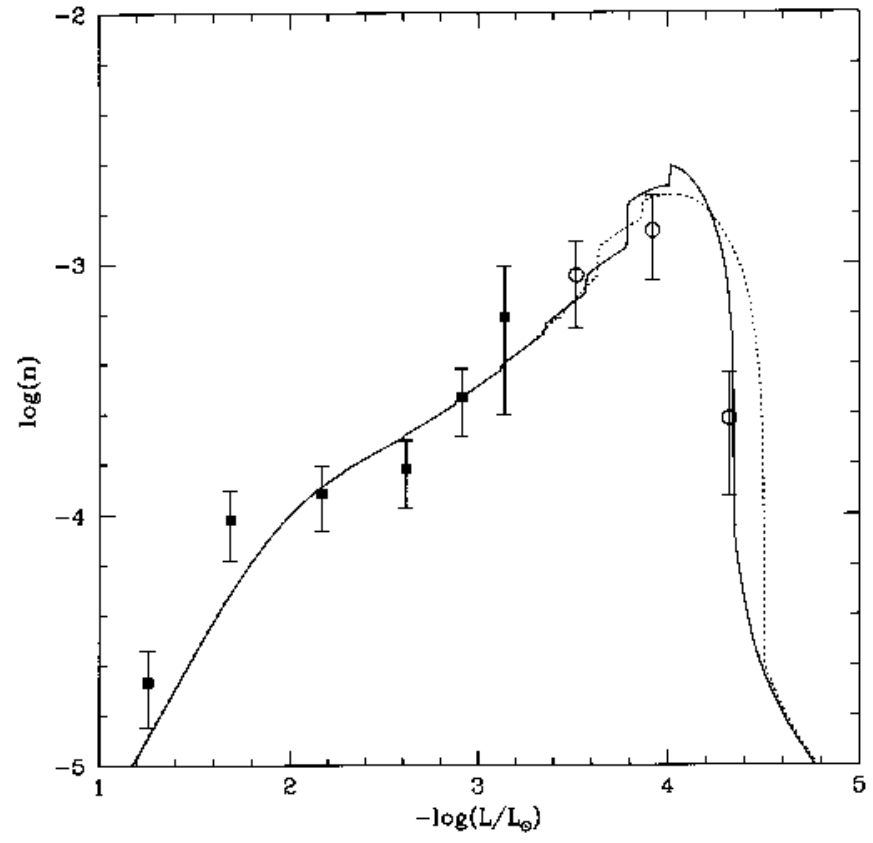

Fig. 4. The white dwarf luminosity function of CO white dwarfs assuming a flat initial profile (solid line) and a stratified initial profile (dotted line) of the carbon-oxygen binary mixture and an age of the disk of 10.5 Gyr. The observational data have been taken from Liebert et al. (1988)

emission rates of Itoh et al. (1992). The computed luminosity function shows a series of steps that are due to the poor resolution in mass. The locations of these steps are associated to the onset of crystallization and phase separation at the center of the star for the succesive masses of our cooling sequences. These steps are smeared out when a finer grid of cooling sequences is adopted. The bump at $\log \left(L / L_{\odot}\right) \approx-4$ is due to the release of gravitational energy caused by chemical fractionation. Finally, the cut-off of the computed white dwarf luminosity function is produced by assuming a finite age of the solar neighborhood. The location of the cut-off is not affected by the size of the adopted grid of the cooling sequences. Since the amount of carbon in the central regions of white dwarfs is larger when flat initial profiles are assumed, the release of gravitational energy due to phase separation is larger and therefore the cut-off of the white dwarf luminosity function is shifted towards higher luminosities. For the same reason the amplitude of the bump at $\log \left(L / L_{\odot}\right) \approx-4$ is larger when flat initial profiles are adopted.

Table 6 displays for comparison $t_{\text {cool }}$ and $\tau_{\text {cool }}$ for a $0.6 M_{\odot}$ when the initial chemical profile is stratified Cols. 2 and 3 - and the same quantities when the initial chemical profile is flat - Cols. 4 and 5. Detailed cooling sequences for white dwarfs of any mass can be obtained on computer readable form upon request to the authors. 
Table 6. Cooling sequences for a $0.6 M_{\odot}$ white dwarf, assuming previous stratification (Cols. 2 and 3) and initial flat profiles (Cols. 4 and 5). The units of $t_{\text {cool }}$ and $\tau_{\text {cool }}$ are Gyr and years, respectively

\begin{tabular}{|c|c|c|c|c|}
\hline $\log \left(L / L_{\odot}\right)$ & $t_{\text {cool }}$ & $\log \left(\tau_{\text {cool }}\right)$ & $t_{\text {cool }}$ & $\log \left(\tau_{\text {cool }}\right)$ \\
\hline-1.0 & 0.000 & 6.866 & 0.000 & 6.874 \\
\hline-1.1 & 0.003 & 6.994 & 0.003 & 7.002 \\
\hline-1.2 & 0.005 & 7.128 & 0.005 & 7.137 \\
\hline-1.3 & 0.008 & 7.269 & 0.008 & 7.278 \\
\hline-1.4 & 0.012 & 7.416 & 0.012 & 7.425 \\
\hline-1.5 & 0.018 & 7.565 & 0.019 & 7.575 \\
\hline-1.6 & 0.027 & 7.712 & 0.028 & 7.723 \\
\hline-1.7 & 0.041 & 7.853 & 0.041 & 7.864 \\
\hline-1.8 & 0.059 & 7.982 & 0.061 & 7.993 \\
\hline-1.9 & 0.085 & 8.093 & 0.087 & 8.105 \\
\hline-2.0 & 0.118 & 8.186 & 0.121 & 8.199 \\
\hline-2.1 & 0.160 & 8.261 & 0.164 & 8.274 \\
\hline-2.2 & 0.208 & 8.322 & 0.214 & 8.335 \\
\hline-2.3 & 0.264 & 8.372 & 0.272 & 8.386 \\
\hline-2.4 & 0.326 & 8.417 & 0.336 & 8.432 \\
\hline-2.5 & 0.395 & 8.460 & 0.407 & 8.475 \\
\hline-2.6 & 0.471 & 8.503 & 0.486 & 8.518 \\
\hline-2.7 & 0.555 & 8.547 & 0.573 & 8.562 \\
\hline-2.8 & 0.649 & 8.593 & 0.670 & 8.609 \\
\hline-2.9 & 0.753 & 8.643 & 0.779 & 8.659 \\
\hline-3.0 & 0.871 & 8.695 & 0.901 & 8.712 \\
\hline-3.1 & 1.004 & 8.751 & 1.039 & 8.768 \\
\hline-3.2 & 1.156 & 8.809 & 1.197 & 8.826 \\
\hline-3.3 & 1.330 & 8.871 & 1.378 & 8.888 \\
\hline-3.4 & 1.532 & 8.935 & 1.588 & 8.952 \\
\hline-3.5 & 1.766 & 9.002 & 1.832 & 9.020 \\
\hline-3.6 & 2.041 & 9.072 & 2.118 & 9.090 \\
\hline-3.7 & 2.556 & 9.402 & 2.455 & 9.162 \\
\hline-3.8 & 3.232 & 9.456 & 2.941 & 9.621 \\
\hline-3.9 & 4.001 & 9.513 & 4.089 & 9.690 \\
\hline-4.0 & 4.878 & 9.567 & 5.390 & 9.731 \\
\hline-4.1 & 5.858 & 9.608 & 6.776 & 9.745 \\
\hline-4.2 & 6.902 & 9.621 & 8.148 & 9.722 \\
\hline-4.3 & 7.934 & 9.597 & 9.396 & 9.662 \\
\hline-4.4 & 8.880 & 9.546 & 10.450 & 9.578 \\
\hline-4.5 & 9.714 & 9.489 & 11.315 & 9.492 \\
\hline-4.6 & 10.452 & 9.440 & 12.038 & 9.421 \\
\hline-4.7 & 11.127 & 9.406 & 12.668 & 9.369 \\
\hline
\end{tabular}

\section{Conclusions}

We have developed a simple method to derive theoretical white dwarf luminosity functions. This method is based on analytical expressions for the relevant quantities of the cooling of white dwarfs such as the cooling times and the characteristic cooling times. These quantities can bederived from fits to the binding energy, once a relationship between the core temperature and luminosity is provided. We have computed binding energies with the most up to date equation of state available, taking into account all the physical processes related to solidification, and in particular chemical fractionation. We have also computed neutrino luminosities using the most recent neutrino rates available. The relationship between the internal temperature and luminosity can be obtained from detailed evolutionary models published elsewhere, which of course depend on the adopted envelopes. Here we have computed white dwarf luminosity functions for two cases of initial chemical profiles and a non-DA model envelope as an example to illustrate the use of the method. Our procedure avoids the developement of a true stellar evolution code, and thus provides a simple, powerful tool to use the white dwarf luminosity function to investigate galactic properties.

Acknowledgements. This work has been supported by DGICYT grants PB94-0827-C02-02 and PB94-0111, by the CESCA Consortium, by the CIRIT and by the AIHF 95-335.

\section{References}

Beaudet G., Petrosian V., Salpeter E.E., 1967, ApJ 150, 979

Chabrier G., 1993, ApJ 414, 695

De Zotti G., 1972, Mem. Soc. Astron. Italiana 43, 89

Díaz-Pinto A., García-Berro E., Hernanz M., Isern J., Mochkovitch R., 1994, A\&A 282, 86

García-Berro E., Hernanz M., Isern J., Mochkovitch R., 1988a, Nat 333, 642

García-Berro E., Hernanz M., Mochkovitch R., Isern J., 1988b, A\&A 193, 141

Hernanz M., García-Berro E., Isern J., Mochkovitch R., Segretain L., Chabrier G., 1994, ApJ 434, 652

Iben I., Tutukov A.V., 1984, ApJ 282, 615

Iben Jr. I., Laughlin G., 1989, ApJ 341, 312

Itoh N., Mutoh H., Hikita A., Kohyama Y., 1992, ApJ 395, 622

Liebert J., Dahn C.C., Monet D.G., 1988, ApJ 332, 891

Lamb D.Q., Van Horn H.M., 1975, ApJ 200, 306

Mazzitelli I., D'Antona F., 1986, ApJ 308, 706

Mochkovitch R., García-Berro E., Hernanz M., Isern J., Panis J.F., 1990, A\&A 233, 456

Noh H.R., Scalo J., 1990, ApJ 352, 605

Segretain L., Chabrier G., 1993, A\&A 271, L13

Segretain L., Chabrier G., Hernanz M., García-Berro E., Isern J., Mochkovitch R., 1994, ApJ 434, 641

Van Horn H.M., 1968, ApJ 151, 227

Weidemann V., Koester D., 1983, A\&A 121, 77

Winget D.E., Hansen C.J., Liebert J., et al., 1987, ApJ 315, L77

Wood M.A., 1990, Ph.D. Thesis, University of Texas at Austin

Wood M.A., 1992, ApJ 387, 662

Wood M.A., Winget D.E., 1989, in White Dwarfs, Wegner G. (ed.). Springer-Verlag, Berlin, 282 